condom was used during last sex act with them. 49\% (231) reported also having non-transactional intimate partners, with whom 46\% (107) reported condomless sex in the last week. $56 \%$ (262) never had an HIV test and $87 \%$ (408) were unaware of HIV prevention programs in Dnipro. HIV prevalence was $1.7 \%(8)$.

Conclusion The high prevalence of HIV-associated vulnerabilities, low testing rates, and high HIV prevalence highlight the importance of designing HIV prevention programs to reach young women engaging in transactional sex.

Disclosure No significant relationships.

\section{P703 PREGNANCY INTENTION AND PREVALENCE ACCORDING TO HIV STATUS AMONG FEMALE SEX WORKERS IN MALI}

${ }^{1}$ Gentiane Perrault Sullivan*, ${ }^{2}$ Nana Camara, ${ }^{3}$ Bintou Dembele, ${ }^{4}$ Fernand Guédou, ${ }^{3}$ Ismaila Thera, ${ }^{5}$ Fatoumata Korika Tounkara, ${ }^{6}$ Michel Alary. ' Laval University, Québec, Canada; ${ }^{2}$ ARCADSIDA, Bamako, Mali; ${ }^{3} A R C A D / S I D A$, Bamako, Mali; ${ }^{4}$ Dispensaire IST, Cotonou, Benin; ${ }^{5}$ Axe Santé des Populations et Pratiques Optimales en Santé, HSS, Social and Preventive Medicine, Québec, Canada; ${ }^{6} \mathrm{CHU}$ de Quebec - Université Laval, Quebec, Canada

\subsection{6/sextrans-2019-sti.769}

Background Women living in Sub-Saharan Africa have the world's highest rates of new HIV infections and unintended pregnancies. These two risks are magnified in the female sex worker's (FSW) population where HIV prevalence is 12 times higher than in the general population. Yet, no information is available concerning FSW's pregnancy intentions, which could help prevent HIV mother-to-child transmission and unintended pregnancies. This study investigated whether pregnancy intention and pregnancy prevalence varied according to FSWs' HIV status. We hypothesized that FSWs living with HIV (FSWLHIV) were less likely to intent carrying a pregnancy and to be pregnant.

Methods We analysed baseline data from a prospective observational cohort study. Three hundreds and twenty-five FSWs were recruited in Bamako, Mali (November 2017 - February 2018). Participants completed a questionnaire. We assessed pregnancy frequencies among women according to HIV status and compared those using chi-square. Age-adjusted odds ratios were estimated using logistic regression.

Results Mean age was 25 years $(\mathrm{N}=303), 20.8 \%$ of the participants were HIV positive and $16.2 \%$ had the intention of becoming pregnant during the next six months. The proportion of FSWs reporting having been pregnant prior to entering in sex work were $72.1 \%(44 / 61)$ for the FSWLHIV and $60.8 \%(142 / 240)$ the other FSWs (p-value=0.2452). The occurrence of pregnancies since engagement in sex work was reported much less often (FSWLHIV $=36.1 \%$ and others $=$ $28.8 \%$ ) with still no significant difference between the two groups ( $\mathrm{p}$-value $=0.3494$ ). FSWLHIV reported more frequently to have wanted a pregnancy in the last 6 months compared to other FSWs (aOR $=2.57,95 \% \mathrm{CI}$ : $[1.3,2.2])$.

Conclusion With FSWLHIV being more likely to desire children during sex work practice, while less than half of them currently receive ART, a specific attention should be given to support them in that decision in order to prevent mother-tochild transmission.

Disclosure No significant relationships.

\section{P705 BACTERIAL SEXUALLY TRANSMITTED INFECTIONS AMONG WOMEN WHO INJECT DRUGS AND EXCHANGE SEX IN KING COUNTY, WASHINGTON}

${ }^{1}$ Megan Curtis, ${ }^{2}$ Courtney Moreno, ${ }^{1}$ Lindley Barbee*, ${ }^{1}$ Sara Glick. ' University of Washington, Medicine, Seattle, USA; ${ }^{2}$ Public Health - Seattle and King County, Seattle, USA

\subsection{6/sextrans-2019-sti.770}

Background Women who inject drugs are at elevated risk for many adverse health outcomes, including sexually transmitted infections (STIs). Exchange sex is prevalent in this population, but its independent effect on STIs is unclear.

Methods We included data regarding the past year from cisgender women age $<60$ who reported injecting drugs and participated in the 2015 or 2016 cycles of the National HIV Behavioral Surveillance (NHBS) survey in Seattle $(\mathrm{N}=377)$. Using a log-binomial regression model, we estimated the odds of self-reported bacterial STI (gonorrhea, syphilis, or chlamydia) among women who inject drugs associated with exchanging sex for money or drugs. We adjusted for sociodemographic characteristics, condom use, and substance use behaviors. We hypothesized that engaging in exchange sex would be independently associated with increased odds of STI.

Results The period prevalence of bacterial STI in the past 12 months was $8.1 \%$ in the full sample. On univariate analysis, STI prevalence was significantly higher among women who reported exchange sex than among those who did not $(10.8 \%$ vs $4.0 \% ; \mathrm{p}=0.02)$ as was STI testing $(53.4 \%$ vs $34.0 \%$; $\mathrm{p}<0.01)$. Women who exchanged sex were less likely to report condomless vaginal or anal sex at last intercourse $(84.4 \%$ vs $89.1 \% ; p=0.19)$. Women who exchanged sex were more likely to use non-injection crack $(59.1 \%$ vs $38.8 \%$; $\mathrm{p}<0.01$ ) and were more likely to report heavy alcohol use (31.3\% vs $19.7 \%$; $=0.01)$. After adjusting for sociodemographic variables, condom use, and substance use; the association between exchange sex and STI remained statistically significant (adjusted odds ratio $=2.7 ; 95 \%$ confidence interval: 1.1-7.2)

Conclusion In this sample of Seattle area women who inject drugs, exchanging sex for drugs or money was associated with double the odds of STI which could be a result of more frequent STI testing. Healthcare providers should prioritize making testing and treatment accessible and appropriate for women who exchange sex.

Disclosure No significant relationships.

\section{P706 LOW GONORRHEA AND CHLAMYDIA TESTING RATE AMONG FEMALE SEX WORKERS IN GUANGDONG PROVINCE: A CROSS-SECTIONAL ANALYSIS}

${ }^{1}$ Peizhen Zhao*, ${ }^{2}$ Fan Yang, ${ }^{1}$ Yajie Wang, 'Weiming Tang, ${ }^{3}$ He-Ping Zheng, ${ }^{4}$ Bin Yang, ${ }^{1}$ Cheng Wang. 'Dermatology Hospital of Southern Medical University, Guangzhou, China; ${ }^{2}$ University of North Carolina Project - China, Guangzhou, China; ${ }^{3}$ Dermatology Hospital of Southern Medical University, Guangdong Center for STD Control and Prevention, Guangzhou, China; ${ }^{4}$ Dermatology Hospital, Southern Medical University, Guangzhou, China

\subsection{6/sextrans-2019-sti.771}

Background Female sex workers (FSW) are at high risk of gonorrhea and chlamydia infection. However, gonorrhea and chlamydia testing rate remain low in this population. This study aimed to assess the gonorrhea and chlamydia testing 